\title{
On the Quantitative Steinitz Theorem in the Plane
}

\author{
P. Brass \\ Fachrichtungen Mathematik - Informatik, Universität Greifswald, \\ Jahnstrasse 15a, D-17489 Greifswald, Germany \\ brass@math-inf.uni-greifswald.d400.de \\ Dedicated to Professor Dr. Jürgen Flachsmeyer on the occasion of his sixtieth birthday
}

\begin{abstract}
We prove that for any $k \geq 4$, any set $X$ of points in the plane, and any point $P \in$ interior $\operatorname{conv}(X)$, there is a subset $Y \subset X$ of at most $k$ points such that if $\operatorname{conv}(X)$ contains a disk with radius $r$ around $P$, then $\operatorname{conv}(Y)$ contains a disk with radius $[\cos (2 /(k+1)) \pi] /[\cos (1 /(k+1)) \pi] r$ around $P$. This generalizes the quantitative Steinitz theorem in the plane and proves a conjecture of Bárány and Heppes.
\end{abstract}

\section{Introduction}

It is a well-known theorem in convexity theory that for any set $X \subset \mathbb{R}^{d}$ and any point $P$ in the interior of $\operatorname{conv}(X)$ there is a subset $Y \subset X$ of at most $2 d$ points such that $P$ is in the interior of conv $(Y)$. This number of $2 d$ points can be necessary, for if $\left(e_{i}\right)_{i=1}^{d}$ is a basis of $\mathbb{R}^{d}$, then $X=\left\{e_{1},-e_{1}, \ldots, e_{d},-e_{d}\right\}$ (the vertexset of a cross-polytope) contains 0 in the interior of its convex hull, but no proper subset of $X$ does.

This theorem of Steinitz was made quantitative by Bárány $e t$ al. [2], who proved the existence of constants $c_{d}>0$ such that if $X \subset \mathbb{R}^{d}$ contains a ball with center $P$ and radius $r$ in its convex hull, then there is a subset $Y \subset X$ of at most $2 d$ points whose convex hull contains a ball with center $P$ and radius $c_{d} r$. Without loss of generality we may always assume that $r=1$ and $P=0$. Bárány $e t$ al. gave a lower bound for $c_{d}$ which is of order $d^{-2 d}$, sketched an improvement to $d^{-(1 / 2) d-2}$, but conjectured the right order of magnitude to be $d^{-1 / 2}$.

Bárány and Heppes [1] determined the constant $c_{2}$ of the quantitative Steinitz theorem in the plane. They proved that any set $X \subset \mathbb{R}^{2}$ whose convex hull contains a disk with center 0 and radius 1 has a subset $Y \subset X$ of at most four points whose convex hull contains a disk with center 0 and radius

$$
c_{2}=\frac{\cos \frac{2}{5} \pi}{\cos \frac{1}{5} \pi}=\frac{3-\sqrt{5}}{2}=0.38 \ldots=: r_{4} \text {. }
$$



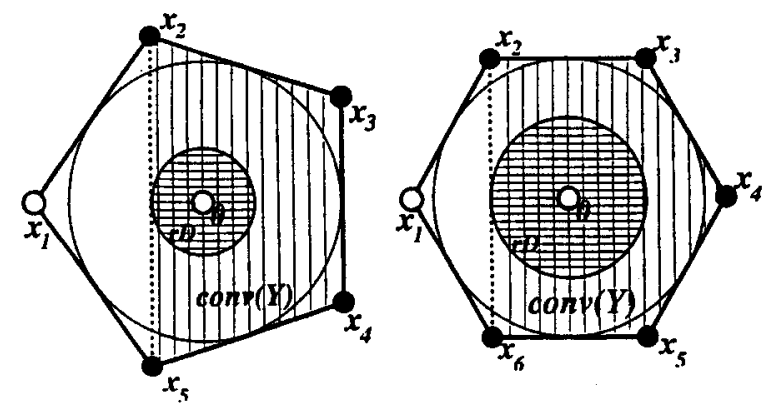

Fig. 1. Extremal situation for four- and five-point subsets.

They also showed the existence of a subset $Y \subset X$ of at most five points whose convex hull contains a disk with center 0 and radius

$$
\frac{\cos \frac{2}{6} \pi}{\cos \frac{1}{6} \pi}=\frac{1}{\sqrt{3}}=0.57 \ldots=: r_{5},
$$

and asked for the largest numbers $r_{k}$ such that $X$ always has a subset $Y \subset X$ of at most $k$ points which contains a disk with center 0 and radius $r_{k}$ in its convex hull conv $(Y)$.

They conjectured that the worst case is always a regular $(k+1)$-gon which contains the unit circle as incircle (as they proved in the cases $k=4,5$, see Fig. 1), so

$$
r_{k}=\frac{\cos (2 /(k+1)) \pi}{\cos (1 /(k+1)) \pi}
$$

It is the aim of this paper to prove that conjecture.

Theorem. If $X \subset \mathbb{R}^{2}$ contains a disk with center 0 and radius 1 in its convex hull $\operatorname{conv}(X)$, then for each $k \geq 4$ there is a subset $Y \subset X$ of at most $k$ points which contains a disk with center 0 and radius $[\cos (2 /(k+1)) \pi] /[\cos (1 /(k+1)) \pi]$ in the convex hull $\operatorname{conv}(Y)$.

These numbers were also investigated by Kirkpatrick et al. [3] for large subset-sizes $k$ and higher dimensions $d$ in connection with a problem in robotics. In the two-dimensional case they had already proved the inequalities

$$
1-2 \frac{\pi^{2}}{k^{2}}<r_{k} \leq \frac{\cos (2 /(k+1)) \pi}{\cos (1 /(k+1)) \pi}<1-\frac{3}{2} \frac{\pi^{2}}{(k+1)^{2}} .
$$

\section{Proof of the Theorem}

Let $D$ be the disk with center 0 and radius 1 . Let a set $X$ in the plane have property $P(r, k)$ if $D \subset \operatorname{conv}(X)$ but for each subset $Y \subset X$ of at most $k$ points $r D \not \subset$ interior conv $(Y)$. Then

$$
r_{k}=\inf \{r \mid \text { there is a set } X \text { with } P(r, k)\} \text {. }
$$


If $X$ has $P(r, k)$ and is an infinite set, then for each $\delta>0$ there is a finite subset $X_{\delta} \subset X$ such that $(1-\delta) D \subset \operatorname{conv}\left(X_{\delta}\right)$. Then $(1-\delta)^{-1} X_{\delta}$ has property $P\left((1-\delta)^{-1} r, k\right)$, therefore

$$
r_{k}=\inf \{r \mid \text { there is a finite set } X \text { with } P(r, k)\} .
$$

The vertices of the regular $(k+1)$-gon which is circumscribed by the disk $D$ give an upper bound

$$
r_{k} \leq r_{k}^{*}:=\frac{\cos (2 /(k+1)) \pi}{\cos (1 /(k+1)) \pi}
$$

for each diagonal from one vertex to the next but one touches the disk $r_{k}^{*} D$ as a tangent, so this set has $P\left(r_{k}^{*}, k\right)$.

We show that $r_{k}=r_{k}^{*}$, i.e., each set $X$ with $D \subset \operatorname{conv}(X)$ has for each $\varepsilon>0$ a subset $Y_{\varepsilon} \subset X$ of at most $k$ points such that $\left(r_{k}^{*}-\varepsilon\right) D \subset$ interior conv $\left(Y_{\varepsilon}\right)$. This implies the theorem; for if $X$ is finite, then there are only finitely many possible choices for $Y_{\varepsilon}$, so there is a $Y$ with $r_{k}^{*} D \subset \operatorname{conv}(Y)$.

Let $r:=\frac{1}{2}\left(r_{k}+r_{k}^{*}\right)$ and let $X$ be a minimum-cardinality set with $P(r, k)$. By (1) $X$ is a finite set. $X$ is the set of vertices of some convex $n$-gon; for if there were a point $x \in X$ with $\operatorname{conv}(X \backslash\{x\})=\operatorname{conv}(X)$, then $x$ could be removed from $X$ without losing $P(r, k)$, a contradiction to the minimum cardinality of $X$. Let $X=\left\{x_{1}, \ldots, x_{n}\right\}$ clockwise, continued $\bmod n$.

Let $\tau: \mathbb{Z} \rightarrow \mathbb{Z}$ be defined by $\tau(i):=\min \left(j \mid j>i\right.$ and $\left.\overline{x_{i} x_{j}} \cap r D \neq \emptyset\right\}$. If $r D$ lies in the interior of the angle $\varangle x_{j} x_{i} x_{j+1}$ for some $j$, then $r D \subset$ interior conv $\left(\left\{x_{i}, x_{j}, x_{j+1}\right\}\right)$, so in that case $X$ would not even have $P(r, 3)$. Therefore there is always such a $j$.

$\tau$ is a monotone increasing mapping, for $i_{1}<i_{2}<\tau\left(i_{2}\right) \leq \tau\left(i_{1}\right)$ implies that $\overline{x_{i_{1}} x_{\tau\left(i_{2}\right)}}$ also intersects $r D$, so $\tau\left(i_{1}\right)=\tau\left(i_{2}\right)$. Since $r<1$ we have $r D \subset$ interior $(D) \subset$ interior $\operatorname{conv}(X)$, so $\tau(i) \geq i+2$ for each $i$. Also since $x_{i+n}=x_{i}$ we have $\tau(i+n)=$ $\tau(i)+n$.

Case 1: $\tau$ is injective. Then $\tau$ is strictly monotone increasing. This implies $\tau(i)=i+a$ for $a:=\tau(1)-1$ and all $i$, since

$$
\tau(i)+n \leq \tau(i+1)+(n-1) \leq \cdots \leq \tau(i+n-1)+1 \leq \tau(i+n)=\tau(i)+n .
$$

Let $l:=\lceil n / a\rceil, n=: a(l-1)+b$, so $1 \leq b \leq a$. Consider the $l$-gon $x_{i}, x_{i+a}, x_{i+2 a}$, $\ldots, x_{i+(l-1) a}$. Since $i+(l-1) a<i+n$ we have

$$
\begin{aligned}
\sum_{\lambda=1}^{l-1}\left|\varangle x_{i+(\lambda-1) a} 0 x_{i+\lambda a}\right| & =2 \pi-\left|\varangle x_{i+(l-1) a} 0 x_{i+n}\right| \\
& =2 \pi-\sum_{\beta=1}^{b}\left|\varangle x_{i+(l-1) a+\beta-1} 0 x_{i+(l-1) a+\beta}\right| \\
& <2 \pi .
\end{aligned}
$$

Taking the average over all $i \in\{1, \ldots, n\}$ and using

$$
\sum_{i=1}^{n} \sum_{\beta=1}^{b}\left|\varangle x_{i+(l-1) a+\beta-1} 0 x_{i+(l-1) a+\beta}\right|=2 \pi b
$$


we find that there is an $\hat{\imath}$ with $\sum_{\beta=1}^{b}\left|\varangle x_{\hat{\imath}+(l-1) a+\beta-1} 0 x_{\hat{\imath}+(l-1) a+\beta}\right| \geq 2 \pi(b / n)$, so

$$
\sum_{\lambda=1}^{l-1}\left|\varangle x_{i+(\lambda-1) a} 0 x_{i+\lambda a}\right| \leq 2 \pi\left(1-\frac{b}{n}\right) .
$$

For each $m \in \mathbb{Z}$ we have $d\left(x_{m}, 0\right) \geq 1, d\left(x_{m+a}, 0\right) \geq 1$ but $d\left(\overline{x_{m} x_{m+a}}, 0\right) \leq r \leq r_{k}^{*}$, therefore $\left|\varangle x_{m} 0 x_{m+a}\right| \geq 2 \arccos (r) \geq 2 \arccos \left(r_{k}^{*}\right)$ holds for each angle $\varangle x_{m} 0 x_{m+a}$. Together this implies $2(l-1) \arccos \left(r_{k}^{*}\right) \leq 2 \pi(1-b / n)$ or

$$
r_{k}^{*} \geq \cos \left(\frac{\pi}{l-1}\left(1-\frac{b}{n}\right)\right)=\cos \left(\pi \frac{a}{a(l-1)+b}\right) .
$$

To get a bound for $k$ in terms of $a, l, n$ we note that

$$
r D \subset \text { interior conv }\left(\left\{x_{a-1}, x_{2(a-1)}, \ldots, x_{[n /(a-1)\rceil(a-1)}\right\}\right),
$$

for by the definition of $\tau$ and $a$ none of the edges of this $\lceil n /(a-1)\rceil$-gon has a nonempty intersection with $r D$. So $X$ does not have property $P(r,\lceil n /(a-1)\rceil)$, therefore

$$
k+1 \leq\left\lceil\frac{n}{a-1}\right\rceil=\left\lceil\frac{a}{a-1}(l-1)+\frac{b}{a-1}\right\rceil .
$$

Using (2) and (3) and the definition and monotonicity of $r_{k}^{*}$ we obtain

$$
\frac{\cos \frac{2 \pi}{\lceil(a /(a-1))(l-1)+b /(a-1)\rceil}}{\cos \frac{\pi}{\lceil(a /(a-1))(l-1)+b /(a-1)\rceil}} \geq \cos \left(\pi \frac{a}{a(l-1)+b}\right) .
$$

Since $(\cos 2 x) /(\cos x)<1-\frac{3}{2} x^{2}$ for $\left.x \in\right] 0, \pi / 2\left[\right.$ and $\cos x>1-\frac{1}{2} x^{2}$ for $x \in[0,1]$ we have

$$
1-\frac{3}{2}\left(\frac{\pi}{\lceil(a /(a-1))(l-1)+b /(a-1)\rceil}\right)^{2}>1-\frac{1}{2}\left(\pi \frac{a}{a(l-1)+b}\right)^{2} .
$$

This implies (using $\lceil(a /(a-1))(l-1)+b /(a-1)\rceil \leq(a /(a-1))(l-1)+$ $(a-2) /(a-1)+b /(a-1))$

$$
\sqrt{3}<\frac{a}{a-1}\left(1+\frac{a-2}{a(l-1)+b}\right)
$$

since $l \geq 3$ and $b \geq 1$ this inequality has no solution with $a \geq 3$. Therefore $a=2$. In this case $X$ has property $P(r, n-1)$. Theorem 3 of [1] states that if a set of $n$ points has $P(r, n-1)$, then $r \geq r_{n-1}^{*}$. Since $\left(r_{v}^{*}\right)_{v=4}^{\infty}$ is a monotonic increasing sequence and $n>k$ 


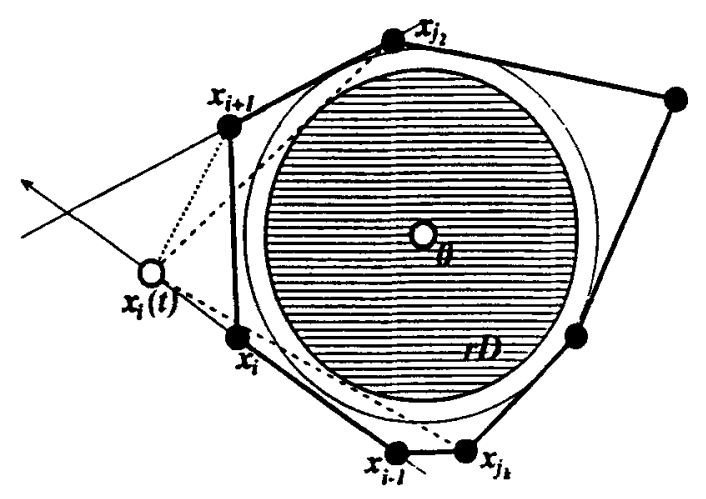

Fig. 2. Situation of Case $2 \mathrm{a}$.

we have $r_{k}^{*} \geq r=\frac{1}{2}\left(r_{k}+r_{k}^{*}\right) \geq r_{n-1}^{*} \geq r_{k}^{*}$. So $n-1=k$ and $r_{k}^{*}=r_{k}$, as the theorem claims.

Case 2: $\tau$ is not injective. Then there is an $i$ with $\tau(i)=\tau(i+1)=: j \geq i+3$. We lead this and the assumption of minimal cardinality of $X$ to a contradiction.

Let $x_{i}(t):=x_{i}+t\left(x_{i}-x_{i-1}\right)$ and $X(t):=\left(X \backslash\left\{x_{i}\right\}\right) \cup\left\{x_{i}(t)\right\}$, so we move $x_{i}$ along the line $x_{i-1} x_{i}$ away from $x_{i-1}$. Then for $t \geq 0$ we have $\operatorname{conv}(X(t)) \supseteq \operatorname{conv}(X) \supset D$.

Case 2a: There is a $t>0$ such that $X(t)$ does not have $P(r, k)$. Then there is a $k$-gon $x_{j_{1}}:=x_{i}(t), x_{j_{2}}, \ldots, x_{j_{k}}$ (clockwise) which completely surrounds $r D$ without touching it, so $r D \subset$ interior conv $\left(\left\{x_{j_{1}}, x_{j_{2}}, \ldots, x_{j_{k}}\right\}\right)$. For each of the finitely many possible $(k-1)$-tuples of indices $\left\{j_{2}, \ldots j_{k}\right\} \subset\{i+1, \ldots, i+n-1\}$ the set $\{t>0 \mid$ $r D \subset$ interior conv $\left.\left(\left\{x_{i}(t), x_{j_{2}}, \ldots, x_{j_{k}}\right\}\right)\right\}$ is open. So if there is a $t>0$ such that $X(t)$ does not have $P(r, k)$, then there is a $t_{0} \geq 0$ such that $X(s)$ has $P(r, k)$ for $s \in\left[0, t_{0}\right]$ but for some $\varepsilon>0$ and each $s \in] t_{0}, t_{0}+\varepsilon$ [ the set $X(s)$ does not have $P(r, k)$. Since only the edges $\overline{x_{i}(t) x_{j_{2}}}$ and $\overline{x_{i}(t) x_{j_{k}}}$ of the $k$-gon $x_{i}(t), x_{j_{2}}, \ldots, x_{j_{k}}$ depend on $t$, this implies that all other edges have empty intersection with $r D$, but at least one of $\overline{x_{i}\left(t_{0}\right) x_{j_{2}}}$ and $\overline{x_{i}\left(t_{0}\right) x_{j_{k}}}$ is a tangent to $r D$ which moves away from $r D$ for $t>t_{0}$ (Fig. 2). $r D$ lies in the angle $\varangle x_{j_{2}} x_{i}\left(t_{0}\right) x_{j_{k}}$. With increasing $t$ the segment $\overline{x_{i}(t) x_{j_{k}}}$ moves toward 0 but the segment $\overline{x_{i}(t) x_{j_{2}}}$ moves away from 0 . This implies that $\overline{x_{i}\left(t_{0}\right) x_{j_{2}}}$ must be the tangent to $r D$ and $\overline{x_{i}\left(t_{0}\right) x_{j k}} \cap r D=\emptyset$. Since $\overline{x_{i}\left(t_{0}\right) x_{j_{2}}} \cap r D \neq \emptyset$ we have $\tau(i) \leq$ $j_{2}$. Furthermore, the segment $\overline{x_{i-1} x_{i}\left(t_{0}\right)}$ does not intersect the line $x_{i+1} x_{j_{2}}$ (otherwise $x_{i+1} \in \operatorname{conv}\left(\left\{x_{i-1}, x_{i}\left(t_{0}\right), x_{j_{2}}\right\}\right)$, so $X\left(t_{0}\right) \backslash\left\{x_{i+1}\right\}$ was a set with $P(r, k)$, contradicting the minimal cardinality of $X$ ). Therefore the line $x_{i}\left(t_{0}\right) x_{j_{2}}$ separates $r D$ and $x_{i+1}$, so it must also separate $x_{i+1}$ and $x_{\tau(i+1)}$. This implies $\tau(i+1)>j_{2} \geq \tau(i)$, a contradiction to the assumption of Case 2.

Case $2 b$ : For each $t \geq 0$ the set $X(t)$ has property $P(r, k)$. Since $X$ is of minimum cardinality, we have for each $t \geq 0$ that $D \not \subset \operatorname{conv}\left(X(t) \backslash\left\{x_{i+1}\right\}\right)$, so for each $t$ the segment $\overline{x_{i}(t) x_{i+2}}$ intersects $D$. Therefore the half-line starting at $x_{i+2}$ in direction $x_{i}-x_{i-1}$ intersects $D$. So the line $g_{1}$ through 0 , perpendicular to $x_{i} x_{i-1}$, separates $x_{i}$ and $x_{i+2}$. 


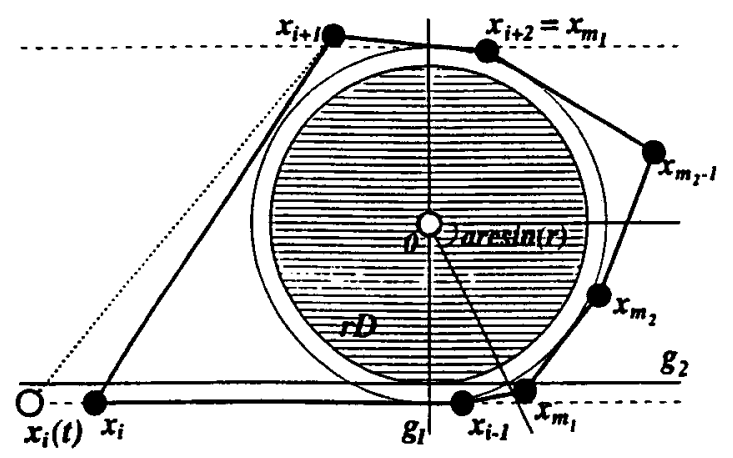

Fig. 3. Situation of Case $2 b$.

Since $\tau(i)=\tau(i+1) \geq i+3$ by the assumption of Case 2 , the segment $\overline{x_{i} x_{i+2}}$ does not intersect $r D$.

Let $g_{2}$ be the parallel to $x_{i} x_{i-1}$ which is a tangent to $r D$ and which separates 0 and $x_{i}$. Any point $x_{m}$ with $i+2 \leq m \leq i+n$ and $\left|\varangle x_{i+2} 0 x_{m}\right| \geq \pi / 2+\arcsin (r)$ lies on the same side of $g_{2}$ as $x_{i}$, so for these points $\overline{x_{m} x_{i}}$ does not intersect $r D$ (Fig. 3).

Let $m_{1}=i+2, m_{v+1}:=\tau\left(m_{v}\right)$ for $v \geq 1$ and let $l$ be the largest $l$ such that $\sum_{\lambda=2}^{l}\left|\varangle x_{m_{\lambda-1}} 0 x_{m_{\lambda}}\right|<\pi / 2+\arcsin (r)$. As in Case 1, each of these angles is at least $2 \arccos (r)$, so we have

$$
2(l-1) \arccos (r)<\frac{\pi}{2}+\arcsin (r) .
$$

If we use now $\arcsin (r) \leq \pi / 2$ we obtain, from (4),

$$
r>\cos \left(\frac{\pi}{2(l-1)}\right) \text {. }
$$

By the definition of $\tau$ we have

$$
r D \subset \text { interior conv }\left(\left\{x_{i}, x_{m_{1}}, x_{m_{2}-1}, x_{m_{2}}, \ldots, x_{m_{l}-1}, x_{m_{l}}, x_{m_{l+1}-1}, x_{m_{l+1}}\right\}\right),
$$

so $k+1 \leq 2(l+1)$. As in Case 1 we now use

$$
r \leq r_{k}^{*} \leq \frac{\cos (2 \pi / 2(l+1))}{\cos (\pi / 2(l+1))}
$$

together with (5) and $\cos (2 x) / \cos (x)<1-\frac{3}{2} x^{2}$ and $\cos (x)>1-\frac{1}{2} x^{2}$ to obtain

$$
\begin{aligned}
1-\frac{3}{2}\left(\frac{\pi}{2(l+1)}\right)^{2} & >\frac{\cos (2 \pi / 2(l+1))}{\cos (\pi / 2(l+1))} \geq r_{k}^{*} \geq r \\
& >\cos \left(\frac{\pi}{2(l-1)}\right)>1-\frac{1}{2}\left(\frac{\pi}{2(l-1)}\right)^{2} .
\end{aligned}
$$

Since the only solutions $l \geq 2$ of $3(1 /(l+1))^{2}<(1 /(l-1))^{2}$ are $l=2,3$, only the cases $l=2, k=4,5$ and $l=3, k=7$ remain. Of these, only the case $l=2, k=5$ 


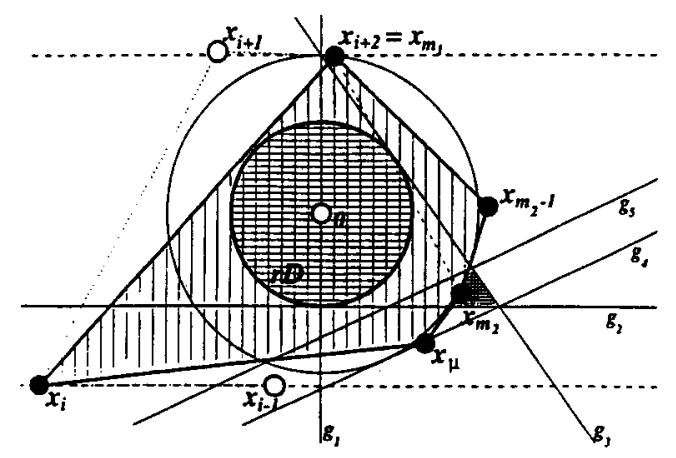

Fig. 4. Situation of Case $2 \mathrm{~b}$ with $l=2, k=5$.

satisfies inequality (4) with $\arccos (r)>\arccos \left(r_{k}^{*}\right), \arcsin (r)<\arcsin \left(r_{k}^{*}\right)$. To exclude this last subcase let $m_{1}, m_{2}$ be as before and let $\mu \geq i+2$ be the first index such that $g_{2}$ separates 0 and $x_{\mu}$ (Fig. 4). We claim that

$$
r D \subset \text { interior } \operatorname{conv}\left(\left\{x_{i}, x_{m_{1}}, x_{m_{2}-1}, x_{m_{2}}, x_{\mu}\right\}\right) .
$$

As before none of the edges $\overline{x_{i} x_{m_{1}}}, \overline{x_{m_{1}} x_{m_{2}-1}}, \overline{x_{m_{2}-1} x_{m_{2}}}$, and $\overline{x_{\mu} x_{i}}$ touches $r D$. If $\mu \leq m_{2}$, this is already sufficient, so we may suppose $\mu-1 \geq m_{2}$. Then $x_{\mu-1}$ and $x_{m_{2}}$ are both in the region bounded by $g_{2}$, the boundary of $D$, and the line $g_{3}$ which is a tangent to $r_{5}^{*} D \supset r D$ through the point of $D$ which is farthest from $x_{i} x_{i-1}$. Since $x_{\mu} x_{\mu-1}$ does not intersect $D$, the tangent $g_{4}$ to $D$ through the point of intersection of $g_{2}$ and $g_{3}$ separates 0 and $x_{\mu}$. Then the parallel $g_{5}$ to $g_{4}$ through the point of intersection of the boundary of $D$ and $g_{3}$ separates $r_{5}^{*} D \supset r D$ and $\overline{x_{\mu} x_{m_{2}}}$. So the set does not have $P\left(r_{5}^{*}, 5\right)$, and Case $2 \mathrm{~b}$ is shown to be impossible.

This completes the proof of the theorem.

\section{References}

1. I. Bárány and A. Heppes. On the exact constant in the quantitative Steinitz theorem in the plane, Discrete Comput. Geom. 12 (1994), 387-398.

2. I. Bárány, M. Katchalski, and J. Pach. Quantitative Helly-type theorems, Proc. Amer. Math. Soc. 86 (1982), 109-114.

3. D. Kirkpatrick, B. Mishra, and C.-K. Yap. Quantitative Steinitz's theorems with applications to multifingered grasping, Discrete Comput. Geom. 7 (1992), 295-318.

Received March 13, 1995. 